Both cases were operated on on Angust 7th, 1900. Chloroform was administered by the house surgeon, Dr. Chalmers. Both proved to be pure meningoceles and the operations offered no points of special interest. The children were sent home after operation and were nursed by their mothers under the directions of one of the sisters. Both healed by first intention, the sutures being removed on the seventh day.

Fig. 3 is a reproduction of a photograph of the patients taken in the early part of October. At that time they were all well, as they are now. They were shown to the Glasgow Medico-Chirurgical Society at a meeting on Nov. 2nd, 1900. region as out-patients after operation and have shown such cases to various medical societies. With properly selected cases I have found the results satisfactory. To insure success selection is less concerned with the infant than with the mother and her circumstances. Given a tiny infant the chances of success in the mother's care, provided she is intelligent and can devote the first few days entirely to nursing under specific instructions, are quite as good as in the wards of an average hospital. The ideal conditions such as may be obtained in private cases include nursing by the mother and by two nurses in addition.

Fig. 3.

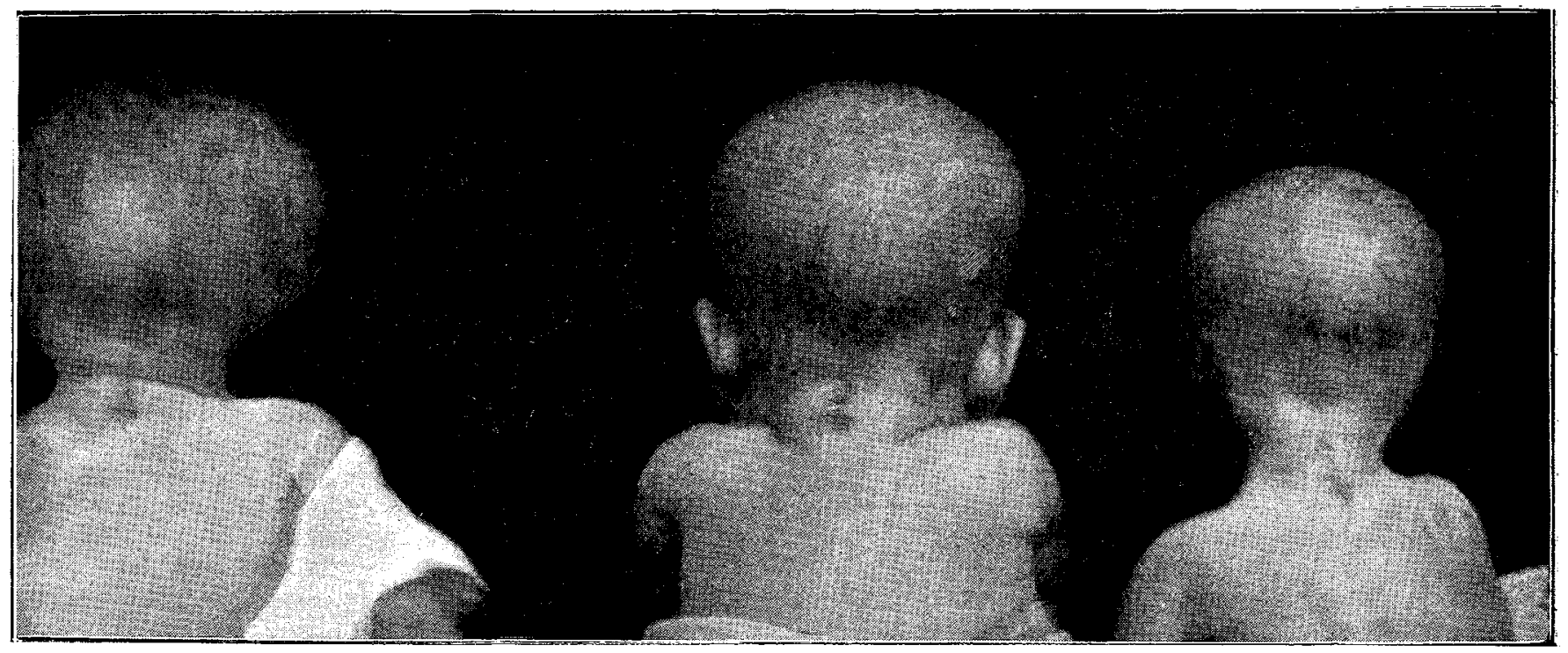

CASE 2.

Case 1.

Case 3.

Photograph of the three cases of cervical spina bifida after excision of the sacs. The patients at this date are aged four months, seven months, and seven months respectively. Operation-Case 1 three and a half months, and Case 2 and Case 3 two months previously.

Remarks. - Spina bifida is not uncommon in the cervical region (though, of course, comparatively much less frequent than in the lumbar region). I have operated on a number of cervical cases, one of which, operated on at the Children's Dispensary in 1896, was, like the three foregoing cases, successfully treated as an out-patient, the child being nursed by the mother under the directions of one of the sisters.

Of all cases of spina bifida, those of the cervical region are the best suited for post-operative treatment as out-patients. I have, however, treated cases of the affection in the lumbar
It is not necessary here to detail the steps of the operation carried out in these three cases. Full accounts of the different methods which I have followed in cases of spina bifida and in cases of hydrocephalus may be found in a paper on the subject in the Glasgow Hospital Reports (vol. ii., 1900), and in an earlier paper read at the meeting of the British Medical Association in Edinburgh and published in the British Medical Journal (Oct. 15th, 1898) and PQdiatrics (1899).

Glasgow.

\section{A CASE OF FRACTURE OF THE STERNUM.}

Br J. SACKVILLE MARTIN, M.D. EdIN., M.R.O.S. ENG.

A SHORT note on this uncommon variety of fracture may be of interest to readers of THE LANCET.

A man, aged 23 years, was working in the pit on Dec. 5 th, 1900. He was leaning forwards, his body bent at right angles to the legs, when a truck which was passing crushed him against a "stack" or upright piece of wood used in keeping up the roof of the gallery. The direction of the force applied was laterally from shoulder to shoulder, and from the fact that his position caused the shoulder to drop forwards the force would naturally act anteriorly to the mid-axillary line. He complained principally of stiffness and soreness about the shoulder-joint and this led me at the time to overlook the condition of the sternum. Five days afterwards, however, I found a very definite projection over the left side of the manubriam sterni ranning vertically downwards. The stiffness of the shoulder had passed off, but any movement which involved bringing the left arm forwards caused pain. On pressing with one finger on the projection and the other on the first rib, crepitus conld be elicited. I was inclined to diagnose fracture of the sternum, but being aware of its rarity, hesitated to do so without a further opinion. Mr. J. E. Platt, surgeon to the
Manchester Royal Infirmary, to whom I sent the patient, confirmed the diagnosis and very kindly wrote to me stating that, in his opinion, the fracture started at the supra-sternal notch and ran downwards and to the left, reaching the edge of the manubrium below the attachment of the first costal cartilage. The detached fragment was somewhat displaced forwards. He also had a skiagram taken, unfortunately with a negative result.

Fractures of the sternum are rare and the form usually described in text-books is a transverse one caused by a heavy weight forcibly flexing the spine and doubling the trunk upon itself. In this case we have a crushing force acting laterally upon the sternum through the line of both clavicles. The clavicles form with the manubrium sterni an arc, and opposing forces applied to both extremities would tend to approximate them and to throw a strain on the centre of the arc. The sudden direct impact of the force through the clavicles combined with this approximation of the arc's extremities probably caused the fracture and would account for the forward displacement of the loose fragment. It is a matter for surprise that the clavicles should have remained uninjured. A fracture of the clavicle, or even a dislocation at the sterno-clavicular joint, would have been a more common result of such an injury.

It is of interest to note the small amount of disanility caused by the fracture. The patient could perform any movements of the left arm five days after the accident and only those associated with the action of the pectoralis major caused him pain. The only treatment adopted was to keep the left arm in a sling, and on Jan 22nd the fracture 
had quite healed and the arm could be used freely without causing him pain. There is, however, a definite ridge, the result of the deformity prodnced by the displacement of the fracture. I am indebted to $\mathrm{Mr}$ Platt for permission to make use of the information he so kindly gave me.

Leigh, Lancashire.

\section{A CASE OF CEREBRO-SPINAL MENINGITIS.}

By FRANCIS RILEY, M.B., B.S. LoND., F.R.C.S. ENG.

IN view of the rarity of sporadic cases of this disease in England it is of some importance that individual cases should be put on record, and the following case presents several points of interest.

A girl, aged five years, who had previously been in good health except that she had been noticed to be lethargic in the mornings, was suddenly attacked with headache and vomiting followed by convulsions. No cause could be assigned except that she had knocked her head against the bedpost in the morning. The same evening the temperature was $103.5^{\circ} \mathrm{F}$., and as it remained about the same

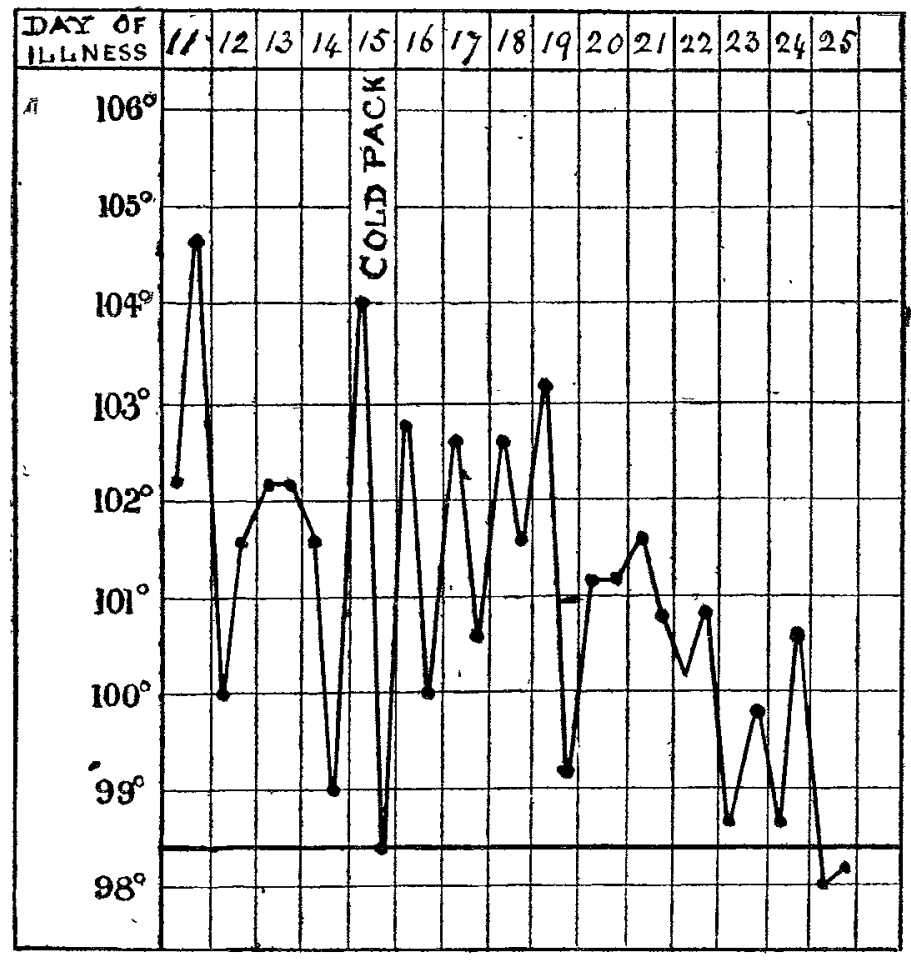

Chart showing the irregular temperature and transition from reversed to normal type.

for several days and all the organs were sound, the suspicion of enteric fever was entertained. On the fourth day it was noticed that the head was retracted and there was slight opisthotonos. The patient was drowsy but answered questions and was irritable when aroused. Both superficial and deep reflexes were abolished except for slight extension of the hallux on plantar stimulation (Babinski's sign). Painful sensation was apparently both diminished and delayed and the tache méningitique was easily obtainable. Only slight headache was complained of after the onset and both pulse and respiration were regular. Sitting up was evidently painful owing to spasm of the cervical muscles, when some difficulty in respiration became manifest. The abdomen was not retracted and no enlargement of the spleen could be made out. The bowels were obstinately confined. Strabismus or facial spasm was absent. On the fifth day a well-marked rash, partly erythematous and partly petechial (the former resembling the rose-spots of enteric fever), was noticed on the trank and extremities and disappeared in the course of a few days. Pain was complained of in one knee and on the dorsum of the foot which was thought to be slightly swollen. On ophthalmoscopic examination the discs were found to be congested, and the pupils reacted slowly to light.

As the case progressed the temperature still remained high-on one occasion reaching $104.6^{\circ}$-and was markedly irregular. During the acute stage the morning reading was considerably higher than that taken in the evening (reversed type), but as the patient began to improve the temperature became lower and showed the normal evening rise. The pulse, regular at first, afterwards became irregular, while the respiration for a short time took on a Cheyne-Stokes type. There were slight ptosis of the left eyelid and photophobia. The reflexes, at first abolished, now became increased, and instead of anæsthesia there was hyperæsthesia. Retraction of the head became more marked, whilst the body was kept in a state of general flexion. There was much irritability, with grinding of the teeth and picking at nose and lips. The child became much emaciated and patches of chloasma appeared on the abdomen and flezor surfaces. The skin over the dorsa and soles of both feet was markedly glossy and shiny owing to the loss of trophic influence (glossy skin of Paget). Occasionally there was vomiting, with a clean tongue, and the urine, which was very dark in colour, was passed unconsciously and smelt very foul.

After about four weeks the patient began to improve, the retraction of the head became less, the pulse and respiration became regular, the hyperæsthesia disappeared, and cutaneous sensibility once again became dulled and the reflexes almost absent. The temperature returned to the ordinary type as convalescence became established and averaged between $99^{\circ}$ and $100^{\circ}$. There were still occasional attacks of vomiting apparently brought on by trifling variations in diet or by sudden changes in the weather, especially when accom. panied by thunderstorms. The total duration of the illness until the patient was able to leave her bed was three months.

The treatment consisted in the application of an icebag to the nape of the neck and during the severity of the fever another was applied to the vertex. A mixture containing bromide and iodide of potassium was given at first to allay irritability and to promote absorption, but as it sometimes provoked vomiting it was not persevered with. As the collapse became more marked small quantities of brandy were given by the mouth and heat was applied to the extremities, and when vomiting interfered with the retention of food rectal enemata were administered. The bowels were kept open by frequent small doses of calomel.

The origin of the illness was obscure, though it is noteworthy that the only other child in the same house contracted diphtheria three weeks after the onset. The surroundings and sanitation appeared to have been good, except for a tub of pig-wash at the rear of the house which was condemned by the medical officer of health. The diagnosis, which was first suspected to be typhoid fever, was placed beyond a doubt by the well-marked spinal symptoms and the appearance of the purpuric eruption which gives the disease the synonym of "spotted fever." Tuberculous meningitis was negatived by the absence of symptoms pointing to an extensive involvement of the cerebral meninges and of any tuberculous focus elsewhere, nor was any history obtainable of a discharge from the nose or ear as a possible cause of purulent meningitis. The presence of Babinski's sign in this disease has not, I think, been before recorded, and the reversed type of temperature is of interest. Herpes labialis, observed so frequently by Tourdes, von Ziemssen, and others, was absent. No definite arthritis, which is so frequently associated with cerebro-spinal meningitis, could be recognised, though pain was complained of in the knee and over the dorsum of one foot, which was thought to be slightly swollen. Headache was conspicuous by its absence, af ter the initial onset being only once or twice complained of. It is remarkable that after the dulness of intellect noticed in the eary stage the mental faculties were preserved throughout and that the untoward sequelæ recorded by other observers, such as various paralyses and eye and ear changes, did not occur, although the emaciation was profound and only gradually recovered from.

Sale, Manchester.

Society of Apothecaries of London. - At the recent examination held by the Society of Apothe cariez for assistants, out of 83 candidates only 31 passed. The examination showed very superficial knowledge on the part of those who failed 